\title{
Dielectric Properties of Polyamides: Polyhexamethylene Adipamide and Polyhexamethylene Sebacamide
}

\author{
Alexander J. Curtis
}

(January 6, 1961)

\begin{abstract}
The dielectric relaxation in two polyamides has been studied over a temperature range from -100 to $175^{\circ} \mathrm{C}$ and a frequency range from $50 \mathrm{c} / \mathrm{s}$ to $10 \mathrm{Mc} / \mathrm{s}$. The effects of various thermal treatments on the relaxation behavior and density, X-ray diffraction, and intrinsic viscosity have been studied. The polyamides were poly(hexamethylene adipamide) and poly(hexamethylene sebacamide). Four relaxation phenomena have been identified. Mechanical relaxation processes are compared with the dielectric phenomena and possible molecular mechanisms are discussed.
\end{abstract}

\section{Introduction}

The dielectric properties of polyamides have received widespread interest in recent years. Boyd $[1]^{1}$ has reported measurements of the dielectric loss of poly(hexamethylene adipamide), 66 nylon, and discussed in detail the high temperature relaxation process in terms of molecular structure. McCall and Anderson have described measurements on a series of seven linear polyamides [2], and Rushton and Russell [13] have studied 66 nylon. The much earlier measurements of the dielectric properties of a number of polyamides by Baker and Yager [3] should also be mentioned.

The polyamides, or as they are commonly called, the nylons, have attracted the attention of a wide group of investigators for a number of reasons. Quite aside from their commercial importance, the nylons are of interest because they possess a highly complex structure and, as expected, have a complicated dielectric relaxation spectrum. The crystalline regions of 66 nylon and of 610 nylon, poly (hexamethylene sebacamide), have been studied by means of X-ray diffraction by Bunn and Garner [4]. It was shown that in the highly ordered crystalline regions, the polymer molecules lie in sheets in which adjacent molecules are joined through hydrogen bonds involving amide protons and carbonyl groups. The two principal lateral spacings determined from $\mathrm{X}$-ray diffraction are, therefore, related to the interchain spacing within the hydrogen-bonded sheets and the intersheet spacing. It has been reported by a number of observers $[5,6,7]$ that these two spacings merge and become indistinguishable above some temperature well below the melting point: in 66 nylon this temperature was reported to be $160{ }^{\circ} \mathrm{C}$ while the crystalline melting point was

1 Figures in brackets indicate the literature references at the end of this paper. observed at $260{ }^{\circ} \mathrm{C}[6]$. The structure above the transition temperature apparently corresponds to a disordered crystal in which the molecules have some rotational freedom about their long axes. It has also been shown that it is possible to obtain samples of nylon in which part or all of the ordered regions are in this rotationally disordered condition at room temperature [7]. This structural feature has been discussed in detail by Sandeman and Keller [8].

The present study of dielectric relaxation in two polyamides, 66 nylon and 610 nylon, is an attempt to correlate dielectric properties with pretreatment variables and in turn to show how these are related to such measurable quantities as crystallinity, moisture content, and molecular weight.

\section{Experimental Details}

\subsection{Dielectric Measurements}

All the dielectric measurements reported here were made with a cell specifically designed for measurements on disk-shaped solids, (fig. 1). All measurements were two-terminal. There is no reason to suspect that the present results, which are consistent with three-terminal measurements on similar materials [1], would have been different if three-terminal measurements had been made.

In figure 1 the cell is shown with the protective enclosure removed. In use, the electrodes were surrounded by this enclosure with heaters built into the walls to provide necessary heating. Temperature control was effected by independent sets of heaters in the top, the sides, and bottom of the enclosure. Thermocouples at the side and top were sensing elements for the controller. Two calibrated chromel-constantan junctions, one below the lower electrode and the other at the upper electrode, served to measure the temperature. The tempera- 


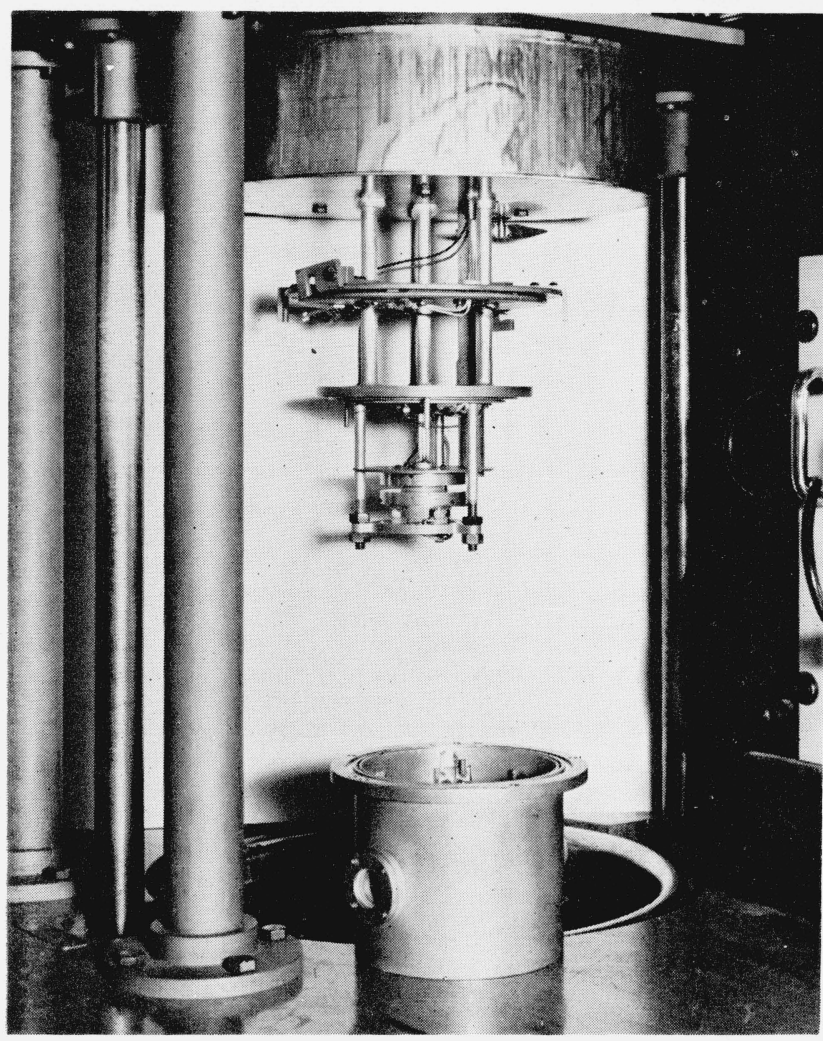

Figure 1. Cell for dielectric measurements shown with sample between electrodes.

ture difference between the upper and lower electrodes was never greater than a tenth of a degree at any of the temperatures at which dielectric measurements were carried out.

Below room temperature the entire cell and enclosure were surrounded by a large Dewar cylinder containing either dry ice or liquid nitrogen, without actually immersing the enclosure in the coolant. The controller supplied whatever current was necessary to maintain the desired temperature. Dry nitrogen was kept flowing through the cell at all times during measurements. At high temperatures, this prevented oxidation of the samples and at low temperatures it prevented condensation of moisture in the system.

Dielectric measurements from $50 \mathrm{c} / \mathrm{s}$ to $100 \mathrm{kc} / \mathrm{s}$ were carried out using a modified General Radio Schering Bridge $(716-\mathrm{C})$. The higher frequency measurements up to $10 \mathrm{Mc} / \mathrm{s}$ were made using a Boonton Q-meter (Type 260-A). The details of the modifications and calibrations will be published elsewhere [9]. The precision and accuracy of the data reported here are in part a function of the dimensions of the samples used. Thus, although capacitance can easily be measured to better than 0.1 percent in almost all cases, the equivalent vacuum capacitance of a sample, as computed from the measured dimensions may involve greater uncertainties, which enter into the computation of each dielectric constant value. In order to reduce probable errors, samples having large equivalent vacuum capacitance were chosen for the low temperature measurements where dielectric constant and loss were relatively small and, correspondingly, samples of small equivalent vacuum capacitance were selected for the high temperature measurements, where extremely large values of dielectric constant and loss were encountered.

For samples having properly selected dimensions, it is estimated that the precision of the measurements on 66 nylon was 0.1 percent in the dielectric constant and 5 percent in the loss index. The measurements on 610 nylon involved the use of a more limited number of samples and similar precision was estimated for all but the low temperature measurements. The errors at low temperatures may have been as great as 1 percent in the dielectric constant, and as large as 10 percent in the loss index.

The principal factor involved in causing errors in measurement of dielectric properties of solids, aside from pretreatment and impurities, is the computation of the equivalent vacuum capacitance of a given disk. The error has been greatly reduced by measuring the diameter of each disk sample with a traveling microscope and computing the thickness from the diameter, the sample weight, and specific volume. This technique which has been described elsewhere [9] has been shown to give equivalent vacuum capacitances to better than 0.1 percent.

\subsection{Preparation and Characterization of Specimens}

The specimens of 66 nylon were pressed from a batch of nylon, in the form of chips, supplied by Dr. Albert Goodman and Miss Helen Anderson of the Du Pont Company. Disk shaped specimens were formed in a special mold designed for this purpose. In this mold, the nylon chips were melted under vacuum and pressed into disks. Because of the large heat capacity of the mold, it was not possible to cool the samples rapidly; generally it took about two hours to cool the mold and sample to room temperature. Because of this unavoidable annealing process, all the 66 nylon samples were quite highly crystalline, as indicated below.

The samples of 610 nylon were supplied by Dr. D. W. McCall of Bell Telephone Laboratories. These samples were from the same lot of polymer used in his work [2], and were supplied as injection molded disks. Some samples for the present study were formed by machining these disks to suitable dimensions. Others were formed by remelting and molding as in the case of the 66 samples.

All the samples were stored in a dessicator over phosphorus pentoxide. Before electrodes were applied, the samples were heated to about $110{ }^{\circ} \mathrm{C}$ in an evacuated container (circa $5-10 \times 10^{-3} \mathrm{~mm}$ ) for three days. Evaporated gold electrodes were applied to most of the samples. Painted silver electrodes were used in some cases. The latter type of electrodes has been found to introduce a very small extraneous dielectric loss in previous studies [23], 
but this effect is certainly negligible in measurements of a material as lossy as the polyamides. In the present study, samples with the two types of electrodes gave identical results. After the electrodes were applied, the samples were again heated to $110{ }^{\circ} \mathrm{C}$ in vacuum for about one day. A variety of further annealing processes were used for the various samples with consequent variations in physical properties, as indicated below.

Specific volume measurements were carried out by a buoyancy technique. The measurements at room temperature and lower were carried out in $n$-heptane, while those at higher temperatures were in a silicone oil bath (Dow Corning "710"). Specific volumes were determined by comparing the measurements with those made simultaneously on a piece of fused silica. The apparatus employed was that described by Hoffman and Weeks [10]. Results obtained at room temperature $\left(23^{\circ} \mathrm{C}\right)$, together with specimen descriptions, are given in table 1 . A typical plot of specific volume as a function of temperature used for the determination of thickness is shown in figure 2 for sample $610-8$. Degrees of crystallinity were computed on the basis of the specific volume scale of Starkweather and Moynihan [11]. It is worth noting that very reproducible specific volumes were obtained for various specimens when nearly identical heat treatments were employed.

Intrinsic viscosities were obtained from measurements on $m$-cresol solutions at $30^{\circ} \mathrm{C}$. These measurements were undertaken to check on possible alterations in molecular weights resulting from the various treatments involved in the preparation of the specimens. The data are listed in table 2, together with sample descriptions. End group analysis gave a number average molecular weight of 16,000 for the 66 nylon chips as received. (Analysis showed 98 moles carboxyl end groups and 38 moles amine end groups per $10^{6} \mathrm{~g}$ of polymer.)

Flory [12] has developed the following relationship between number-average molecular weight (deter- mined by end group analysis) and intrinsic viscosity in $m$-cresol for 66 nylon:

$$
\overline{\mathrm{M}}_{n}=17,800[\eta]-3,300 .
$$

The above expression demonstrates the well-known monotonic relationship between molecular weight and intrinsic viscosity for polymers. Using the

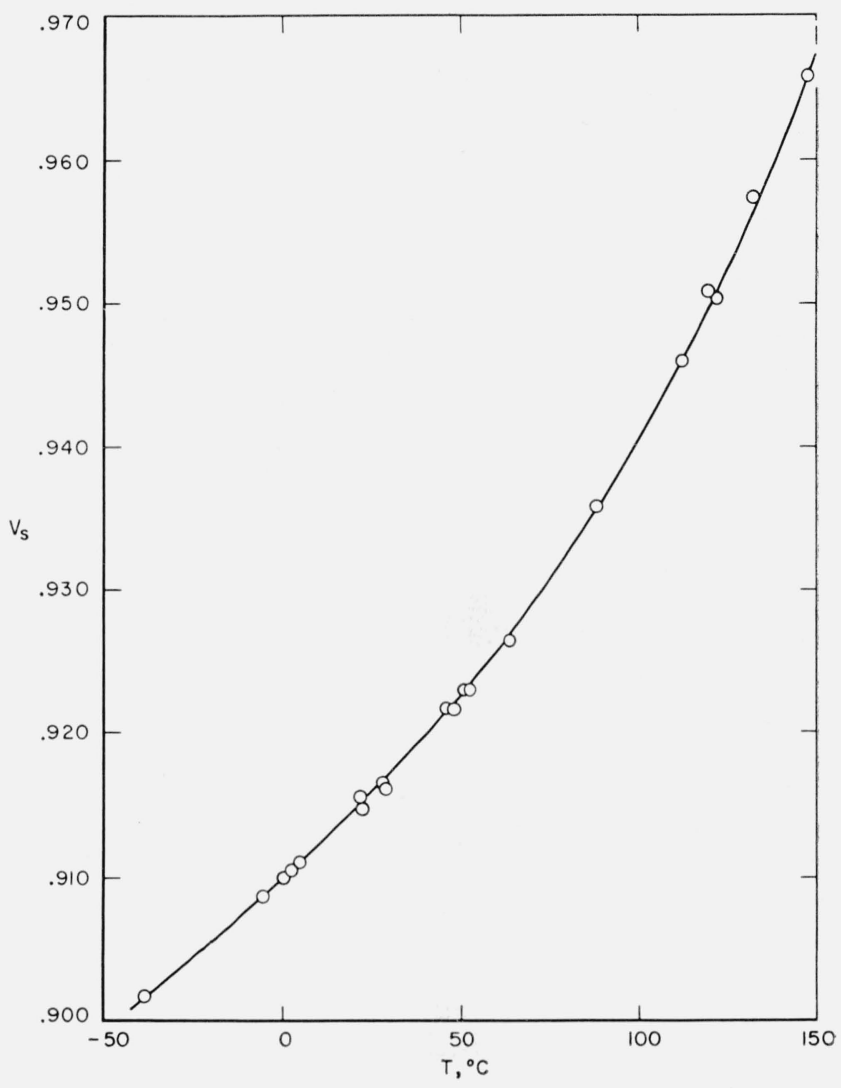

Figure 2. Specific volume $\left(\mathrm{cm}^{3} / \mathrm{g}\right)$ of 610 nylon as a function of of temperature.

TABLE 1. Sample descriptions and specific volumes

\begin{tabular}{|c|c|c|c|c|c|}
\hline Number & Mode of preparation and thermal history & $\begin{array}{l}\text { Specific } \\
\text { volume } \\
\text { at } 23^{\circ} \mathrm{C}\end{array}$ & $\begin{array}{c}\% \text { Crystal- } \\
\text { linity (see } \\
\text { text) }\end{array}$ & Thickness & Area \\
\hline $66-1$ & $\begin{array}{l}\text { Melted in vacuum, compression molded, heated in vacuum for } \\
\text { three days at } 130{ }^{\circ} \mathrm{C} \text {. }\end{array}$ & $\begin{array}{l}c m^{3} / g \\
0.8709\end{array}$ & 56 & 0. $\begin{array}{cc}\mathrm{cm} \\
3540\end{array}$ & $\begin{array}{l}c m^{2} \\
10.372\end{array}$ \\
\hline $66-2$ & Same as $66-1$. & .8637 & 62 & .3823 & 5. 0623 \\
\hline $66-3$ & Same as $66-1$ & .8676 & 58 & 1461 & 10.525 \\
\hline $66-4$ & Cut from same disc as $66-3$ & .8676 & 58 & .1485 & 10.535 \\
\hline $66-6$ & $\begin{array}{l}\text { Melted in vacuum, compression molded. (Heated in vacuum } \\
\text { for subsequent measurements, no perceptible change in } V_{s} \text { ) }\end{array}$ & .8685 & 58 & .3365 & 7. 9118 \\
\hline $610-1$ & $\begin{array}{l}\text { Injection molded, stored one month over } \mathrm{P}_{2} \mathrm{O}_{5} \text {. } \\
\text { After heating in vacuum to } 110^{\circ} \mathrm{C} \text { for three days. } \\
\text { After heating to } 210^{\circ} \mathrm{C} \text { for one day. }\end{array}$ & $\begin{array}{l}9289 \\
9287 \\
9138\end{array}$ & $\begin{array}{l}34 \\
34 \\
50\end{array}$ & .3197 & 7. 285 \\
\hline $610-2$ & Melted in vacuum, heated five days in vacuum at $130^{\circ} \mathrm{C}$. & 9181 & 46 & .2545 & 7. 905 \\
\hline $610-7$ & $\begin{array}{l}\text { Injection molded, stored over } \mathrm{P}_{2} \mathrm{O}_{5} \\
\text { After drying at } 130{ }^{\circ} \mathrm{C} \text { in vacuum for five days. }\end{array}$ & $\begin{array}{l}9289 \\
.9248\end{array}$ & $\begin{array}{l}34 \\
38\end{array}$ & & \\
\hline & After heating in vacuum at $210^{\circ} \mathrm{C}$ for one day. & .9156 & 48 & .2924 & 7. 785 \\
\hline $610-8$ & Melted in vacuum, heated five days at $130^{\circ} \mathrm{C}$ in vacuum. & .9150 & 49 & .1745 & 10. 616 \\
\hline $610-9$ & Injection molded and heated three days at $110^{\circ} \mathrm{C}$. & & & .1578 & 11. 445 \\
\hline
\end{tabular}


intrinsic viscosity value obtained on polymer "as received" from table 2 (1.18), one obtains $\overrightarrow{\mathrm{M}}_{n}=17,700$ which is in fair agreement with the value obtained from end group analysis.

It is evident from the data in table 2 that the various heat treatments carried out to dry the specimens have caused a definite increase in molecular weight. This effect will be dealt with subsequently.

The X-ray diffraction properties of the various samples were also measured with a North American Phillips diffractometer. Sample traces of X-ray intensity as a function of diffraction angle are shown in figure 3. These, and specific volume data, show that high temperature annealing is necessary to obtain maximum crystallinity in these polyamides.

The d-c resistance measurements were made with a Beckman Ultrohmeter with an external $200 \mathrm{v}$ potential for resistances greater than $10^{9}$ ohms. Resistances below this value were measured with a General Radio Type 544-B megohm bridge. In the temperature region where the d-c conduction was of particular interest, i.e., above about $80^{\circ} \mathrm{C}$, it was found that there was such a large drift in resistance after applying the measuring potential that the results were of questionable value and low reproducibility.

TABLE 2. Intrinsic viscosities

In $m$-cresol at $30^{\circ} \mathrm{C}$.

\begin{tabular}{|c|c|}
\hline Sample description & \\
\hline 66 Nylon samples: & \\
\hline $\begin{array}{l}\text { Chips, as received } \\
\text { Chips, dried in vacuum at } 130^{\circ} \mathrm{C} \text { for } 3 \text { days } \\
\text { Vacuum melted, dried in vacuum at } 130^{\circ} \mathrm{C} \text { for } 3 \text { days } \\
\text { Vacuum melted, dried in vacuum at } 130^{\circ} \mathrm{C} \text { for } 3 \text { days }\end{array}$ & $\begin{array}{l}\text { 1. } 18 \\
\text { 1. } 26 \\
\text { 1. } 69 \\
\text { 1. } 73\end{array}$ \\
\hline 610 Nylon samples: & \\
\hline $\begin{array}{l}\text { Injection molded, as received } \\
\text { Injection molded, dried at } 130^{\circ} \mathrm{C} \text { for } 3 \text { days } \\
\text { Melted in vacuum, dried at } 130^{\circ} \mathrm{C} \text { for } 3 \text { days } \\
\text { Melted in vacuum, dried at } 210^{\circ} \mathrm{C} \text { for } 1 \text { day }\end{array}$ & $\begin{array}{r}0.96 \\
.96 \\
\text { 1. } 26 \\
1.33\end{array}$ \\
\hline
\end{tabular}
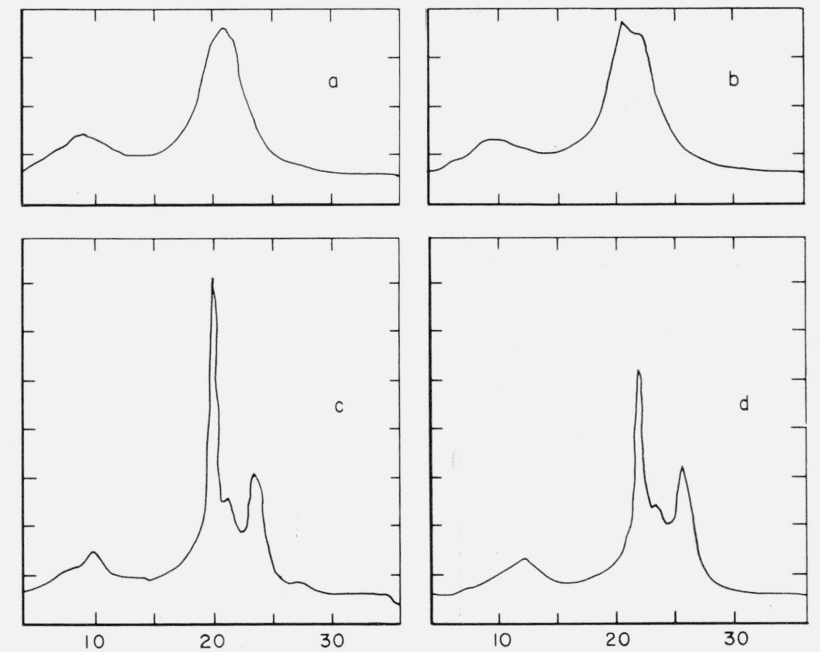

Figure 3. Relative X-ray intensity versus 20, (nickel-filtered copper $K$ radiation). 6-10 Nylon; (A) injection molded, no heat treatment; (B) after five days in
vacuum at $130^{\circ} \mathrm{C}$; (C) melted in vacuum, cooled slowly in mold; (D) injection vacuum at $1300^{\circ} \mathrm{C}$; (C) melted in vacuum,
molded, heated 1 day in vacuum to $210^{\circ} \mathrm{C}$.
The data shown in figure 4 are computed from the only set of measurements made.

\subsection{Experimental Results}

The dielectric constants and losses of the various 66 nylon specimens are listed in table 3. Similar measurements on the 610 nylon samples are given in table 4. Thermal history of each specimen is briefly described in the tables.

There are several cases of nearly identical samples. The differences in the dielectric properties of these samples reflect the difficulty of obtaining semicrystalline polymer samples in identical physical states: for example, nylon specimen 610-1, after drying at $210^{\circ} \mathrm{C}$, had $\epsilon^{\prime}=8.311$ and $\epsilon^{\prime \prime}=0.950$ while specimen $610-7$, given similar treatment had $\epsilon^{\prime}=8.391$ and $\epsilon^{\prime \prime}=0.984$, all measured at $1 \mathrm{kc} / \mathrm{s}$ and $80^{\circ} \mathrm{C}$.

The data in table 3 have not been corrected for the effect of d-c conduction on observed dielectric loss. As noted above, in the temperature region in which this correction would become significant, the measurement of $d$-c resistance becomes somewhat arbitrary. The significance of $\mathrm{d}$-c conduction

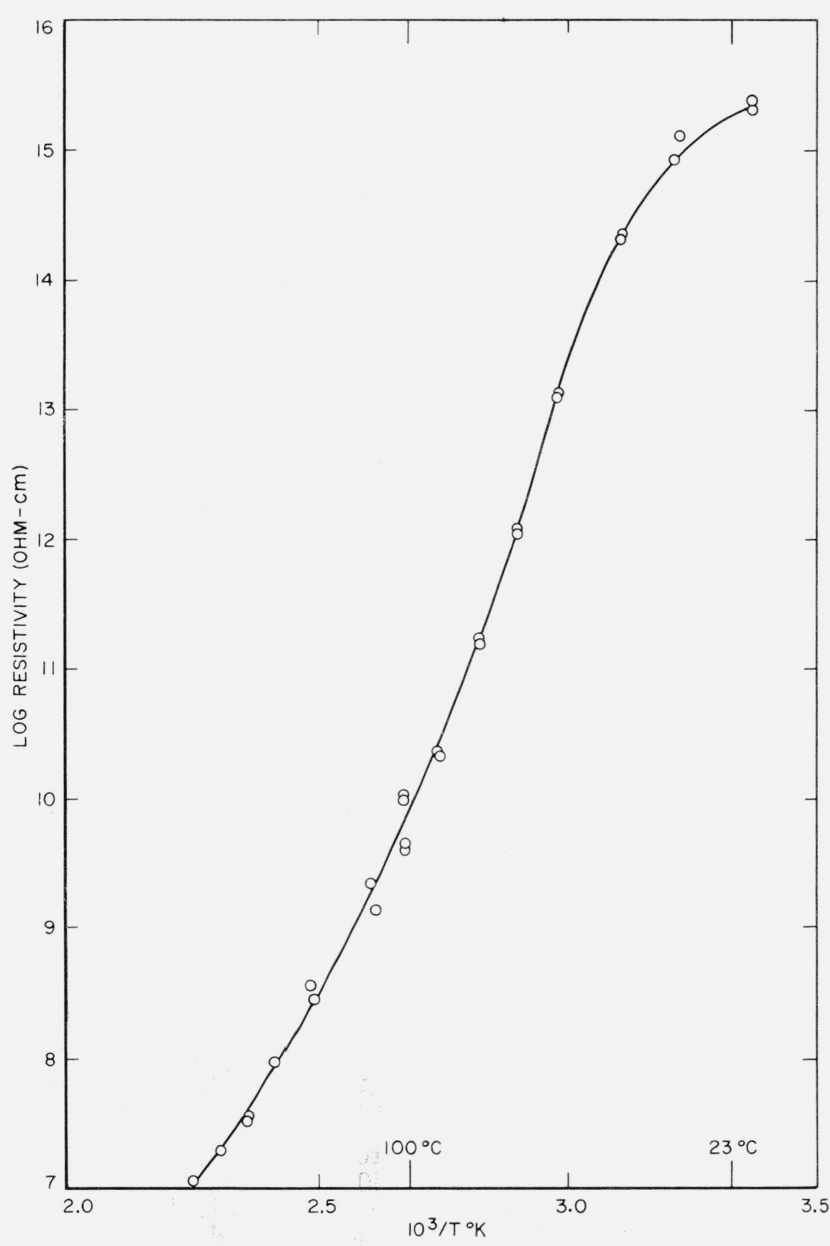

FIgURE 4. D-C resistivity of 66 nylon as a function of the reciprocal temperature. 
TABLE 3. Dielectric constants and loss indexes of 66 nylon samples

\begin{tabular}{|c|c|c|c|c|c|c|c|c|c|c|c|c|}
\hline \multirow{7}{*}{$\begin{array}{c}\text { Frequency } \\
50 \mathrm{c} / \mathrm{s} \\
100 \\
200 \\
500\end{array}$} & \multicolumn{12}{|c|}{$66-1$} \\
\hline & \multicolumn{2}{|c|}{$23^{\circ} \mathrm{C}$} & \multicolumn{2}{|c|}{$51.3^{\circ} \mathrm{C}$} & \multicolumn{2}{|c|}{$75.2^{\circ} \mathrm{C}$} & \multicolumn{2}{|c|}{$99^{\circ} \mathrm{C}$} & \multicolumn{2}{|c|}{$125.4^{\circ} \mathrm{C}$} & \multicolumn{2}{|c|}{$149.6^{\circ} \mathrm{C}$} \\
\hline & & $\epsilon^{\prime \prime} \times 10^{4}$ & $\epsilon^{\prime}$ & $\epsilon^{\prime \prime} \times 10^{4}$ & $\epsilon^{\prime}$ & $\epsilon^{\prime \prime}$ & $\epsilon^{\prime}$ & $\epsilon^{\prime \prime}$ & $\epsilon^{\prime}$ & $\epsilon^{\prime \prime}$ & $\epsilon^{\prime}$ & $\epsilon^{\prime \prime}$ \\
\hline & 3. 570 & 278. 7 & 3. 926 & 842 & 7. 526 & 1. 969 & & & & & & \\
\hline & 3. 560 & 327.4 & 3. 900 & 771 & 6. 961 & 1. 529 & 20.56 & 19. 04 & & & & \\
\hline & $\begin{array}{l}3.546 \\
3.522\end{array}$ & $\begin{array}{r}346.2 \\
397.6\end{array}$ & 3. 873 & $\begin{array}{r}685 \\
666\end{array}$ & 6. 487 & 1. 101 & 16. 94 & 11.77 & 50.72 & 100.1 & & \\
\hline & $\begin{array}{l}3.522 \\
3.505\end{array}$ & $\begin{array}{l}397.6 \\
421\end{array}$ & $\begin{array}{l}3.834 \\
3.800\end{array}$ & $\begin{array}{r}666 \\
683\end{array}$ & 5. 991 & 0.8045 & $\begin{array}{l}13.27 \\
11.85\end{array}$ & $\begin{array}{l}6.49 \\
4.30\end{array}$ & & & & 140.1 \\
\hline $2^{1 \mathrm{KC} / \mathrm{s}}$ & 3. 486 & 458 & 3. 780 & 714 & $\begin{array}{l}5.728 \\
5.495\end{array}$ & $\begin{array}{l}.6765 \\
.584\end{array}$ & $\begin{array}{l}11.85 \\
10.91\end{array}$ & $\begin{array}{l}4.30 \\
2.96\end{array}$ & 26.78 & $\begin{array}{l}29.38 \\
18.69\end{array}$ & $\begin{array}{l}60.32 \\
43.62\end{array}$ & $\begin{array}{r}140.1 \\
82.0\end{array}$ \\
\hline $\begin{array}{l}2 \\
5\end{array}$ & $\begin{array}{l}\text { 0. } 400 \\
3.455\end{array}$ & $\begin{array}{l}400 \\
499\end{array}$ & 3. 733 & 795 & 5. 186 & $\begin{array}{l}.084 \\
.499\end{array}$ & $\begin{array}{r}10.91 \\
9.82\end{array}$ & $\begin{array}{l}2.90 \\
1.95\end{array}$ & 16.44 & $\begin{array}{r}18.00 \\
9.78\end{array}$ & $\begin{array}{l}40.02 \\
30.55\end{array}$ & $\begin{array}{l}8.0 \\
39.7\end{array}$ \\
\hline 10 & 3. 432 & 514 & 3. 698 & 841 & 4. 987 & 455 & 9.24 & 1. 60 & 14.55 & 6.118 & 24.82 & 24.3 \\
\hline 20 & 3. 411 & 516 & 3. 661 & 865 & 4. 810 & .428 & 8. 717 & 1. 47 & 13.44 & 4. 035 & 19.31 & 14.92 \\
\hline 50 & 3. 376 & 541 & 3. 664 & 919 & 4. 550 & .393 & 7. 881 & 1. 34 & 12. 22 & 2. 445 & 15. 89 & 7. 66 \\
\hline 100 & 3. 352 & 566 & 3. 563 & 948 & 4. 389 & .374 & 7. 315 & 1. 34 & 11. 63 & 1. 976 & 14.51 & 4. 79 \\
\hline 310 & 3. 292 & 570 & 3. 493 & 902 & 4. 144 & .2959 & 6.516 & 1. 102 & 10.75 & 1. 496 & & \\
\hline $1 \mathrm{Mc} / \mathrm{s}$ & 3. 261 & 612 & 3. 436 & 901 & 3. 963 & .2560 & 5. 558 & 0.953 & 9. 43 & 1. 603 & & \\
\hline 3.2 & 3. 211 & 647 & 3. 374 & 888 & 3. 743 & $\begin{array}{l}.2000 \\
.2200\end{array}$ & 4. 817 & $\begin{array}{r}0.900 \\
.750\end{array}$ & $\begin{array}{l}9.40 \\
7.79\end{array}$ & $\begin{array}{l}1.000 \\
1.678\end{array}$ & & \\
\hline 10 & 3. 165 & 708 & 3. 305 & 921 & 3. 593 & .2041 & 4. 313 & .593 & 6.19 & 1. 505 & & \\
\hline
\end{tabular}

\begin{tabular}{|c|c|c|c|c|c|c|c|c|c|c|}
\hline \multirow{4}{*}{$\begin{array}{l}\text { Frequency } \\
50 \mathrm{c} / \mathrm{s}\end{array}$} & \multicolumn{10}{|c|}{$66-2$} \\
\hline & \multicolumn{2}{|c|}{$23.5^{\circ} \mathrm{C}$} & \multicolumn{2}{|c|}{$51.3^{\circ} \mathrm{C}$} & \multicolumn{2}{|c|}{$61.4^{\circ} \mathrm{C}$} & \multicolumn{2}{|c|}{$66.0^{\circ} \mathrm{C}$} & \multicolumn{2}{|c|}{$71.3^{\circ} \mathrm{C}$} \\
\hline & & $\epsilon^{\prime \prime} \times 10^{4}$ & & $\epsilon^{\prime \prime} \times 10^{4}$ & & $\epsilon^{\prime \prime} \times 10^{4}$ & & $\epsilon^{\prime \prime} \times 10^{4}$ & $\epsilon^{\prime}$ & $\epsilon^{\prime \prime} \times 10^{4}$ \\
\hline & 3. 641 & 328 & 3. 956 & 840 & 4. 538 & 3120 & 5.067 & 5600 & 5. 770 & 9330 \\
\hline 100 & 3. 627 & 365 & 3. 931 & 763 & 4. 441 & 2590 & 4. 892 & 4560 & 5. 491 & 7290 \\
\hline 200 & 3. 612 & 372 & 3.910 & 716 & 4. 357 & 2040 & 4. 754 & 3540 & 5. 265 & 5570 \\
\hline 500 & 3.587 & 401 & 3. 874 & 699 & 4. 249 & 1670 & 4. 575 & 2760 & 4. 979 & 4170 \\
\hline $1 \mathrm{kc} / \mathrm{s}$ & 3. 572 & 438 & 3.845 & 709 & 4. 187 & 1500 & 4. 474 & 2400 & 4. 826 & 3510 \\
\hline 2 & 3. 549 & 451 & 3.817 & 755 & 4. 130 & 1430 & 4. 388 & 2160 & 4. 700 & 3120 \\
\hline 5 & 3. 516 & 480 & 3. 770 & 801 & 4. 044 & 1390 & 4. 262 & 1990 & 4. 524 & 2750 \\
\hline 10 & 3. 494 & 496 & 3. 735 & 827 & 3. 985 & 1380 & 4. 181 & 1890 & 4. 416 & 2580 \\
\hline 20 & 3,473 & 476 & 3.700 & 846 & 3. 928 & 1350 & 4. 106 & 1860 & 4. 316 & 2460 \\
\hline 50 & 3. 441 & 499 & 3. 642 & 859 & 3. 838 & 1330 & 3.985 & 1790 & 4. 162 & 2320 \\
\hline 100 & 3.418 & 529 & 3. 608 & 885 & 3. 776 & 1340 & 3. 905 & 1770 & 4.065 & 2260 \\
\hline 310 & 3. 293 & 526 & 3.481 & 851 & 3. 635 & 1240 & 3. 693 & 1620 & 3.868 & 1980 \\
\hline $1 \mathrm{Mc} / \mathrm{s}$ & 3. 276 & 534 & 3.455 & 850 & 3. 559 & 1180 & 3. 625 & 1440 & 3. 749 & 1820 \\
\hline 3. 2 & 3. 259 & 584 & 3.404 & 858 & 3. 491 & 1120 & 3.574 & 1330 & 3. 605 & 1610 \\
\hline 10 & 3. 191 & 683 & 3. 303 & 962 & 3. 389 & 1170 & 3. 438 & 1300 & 3.503 & 1540 \\
\hline
\end{tabular}

\begin{tabular}{|c|c|c|c|c|c|c|c|c|c|c|c|c|}
\hline \multirow{7}{*}{$\begin{array}{l}\text { Frequency } \\
50 \mathrm{c} / \mathrm{s} \\
100 \\
200 \\
500\end{array}$} & \multicolumn{12}{|c|}{ 66-2 (Cont.) } \\
\hline & \multicolumn{2}{|c|}{$75.8^{\circ} \mathrm{C}$} & \multicolumn{2}{|c|}{$80.0^{\circ} \mathrm{C}$} & \multicolumn{2}{|c|}{$99.8^{\circ} \mathrm{C}$} & \multicolumn{2}{|c|}{$125.9^{\circ} \mathrm{C}$} & \multicolumn{2}{|c|}{$150.2^{\circ} \mathrm{C}$} & \multicolumn{2}{|c|}{$175.2^{\circ} \mathrm{C}$} \\
\hline & $\epsilon^{\prime}$ & $\epsilon^{\prime \prime}$ & $\epsilon^{\prime}$ & $\epsilon^{\prime \prime}$ & $\epsilon^{\prime}$ & $\epsilon^{\prime \prime}$ & $\epsilon^{\prime}$ & $\epsilon^{\prime \prime}$ & $\epsilon^{\prime}$ & $\epsilon^{\prime \prime}$ & $\epsilon^{\prime}$ & $\epsilon^{\prime \prime}$ \\
\hline & 6. 989 & 1. 677 & 8. 497 & 2. 798 & 22. 99 & 23.76 & & & & & & \\
\hline & 6.508 & 1. 253 & 7. 698 & 2. 052 & 18.60 & 15. 29 & 40.64 & 168.3 & & & & \\
\hline & 6. 137 & 0.9413 & 7. 122 & 1. 456 & 15. 24 & 9. 962 & 36.44 & 89.13 & & & & \\
\hline & 5. 694 & .6750 & 6. 468 & 0.997 & 12. 284 & 5. 920 & 28.73 & 44. 42 & 44.68 & 262.62 & & \\
\hline $1 \mathrm{kc} / \mathrm{s}$ & 5. 461 & .5642 & 6.135 & .803 & 10.828 & 3. 902 & 23. 70 & 26.40 & 37.67 & 138.23 & & \\
\hline 2 & 5. 270 & 4963 & 5.862 & 690 & 9.857 & 2. 659 & 19. 297 & 16. 61 & 32.57 & 74. 09 & 41.35 & 322.31 \\
\hline 5 & 4. 992 & .4257 & 5.477 & .577 & 8. 992 & 1. 767 & 15. 199 & 9. 157 & 26.21 & 35.81 & 35.05 & 135. 22 \\
\hline 10 & 4. 827 & .3862 & 5. 243 & .5228 & 8. 461 & 1. 421 & 13. 288 & 5. 692 & 21. 447 & 21.63 & 30.86 & 73.47 \\
\hline 20 & 4. 682 & 3645 & 5.042 & .4847 & 7. 969 & 1. 250 & 12.061 & 3. 667 & 17. 647 & 13. 210 & 26.15 & 40.71 \\
\hline 50 & 4. 459 & 3361 & 4.745 & 4399 & 7.150 & 1. 204 & 11.082 & $\begin{array}{l}0.223 \\
2.223\end{array}$ & 14. 461 & 7. 053 & 17.98 & 18.87 \\
\hline 100 & 4. 320 & 3193 & 4. 562 & 4120 & 6.535 & 1.009 & 10.510 & 1. 756 & 12.804 & 4.334 & 16. 80 & 12.55 \\
\hline 310 & 4. 087 & .2735 & 4. 245 & .3526 & 6.13 & 1.030 & 9.80 & 1. 513 & 12.15 & $\begin{array}{l}\text { t. } 14 t \\
\text { 2. } 141\end{array}$ & 13.90 & 5. 295 \\
\hline $1 \mathrm{Mc} / \mathrm{s}$ & 3. 909 & .2411 & 4. 008 & .2968 & 5. 37 & 0.868 & 8.63 & 1. 623 & 11. 23 & 1. 572 & 12.63 & 2. 320 \\
\hline $\begin{array}{l}\text { 1. } 2 \\
\text { - }\end{array}$ & 3. 663 & 2073 & 3. 779 & .2467 & 4. 70 & $\begin{array}{r}.079 \\
.679\end{array}$ & 7. 41 & 1. 656 & 10. 24 & 1. 704 & 11.94 & 1. 501 \\
\hline 10 & 3. 570 & .1839 & 3. 601 & .2181 & 4. 18 & .522 & 5.97 & 1. 497 & 8.87 & 2. 091 & 11.07 & 1. 726 \\
\hline
\end{tabular}

will be discussed below. In this particular case, we prefer not to alter the data in an arbitrary manner by using an assumed value of the d-c conduction.

\section{Discussion of Results}

The important features of the dielectric relaxation behavior of the two polyamides examined in this study may be summarized as follows:

(1) Both polymers exhibit a relaxation phenomenon which appears as a loss maximum at temperatures above about $80^{\circ} \mathrm{C}$ and at frequencies above $1 \mathrm{kc} / \mathrm{s}$. Some of the data from tables 3 and 4 are plotted in figures 5 and 6 . It is seen that the relaxa- tion process is similar in the two materials. This is the same relaxation effect identified as the " $\alpha$ " process by Boyd [1] and studied in detail by him in 66 nylon. It is also the same as that studied by McCall and Anderson [2] in 66 nylon as well as in six other polyamides.

(2) Above about $50{ }^{\circ} \mathrm{C}$, both polymers exhibit a low frequency polarization as manifested by a large dielectric constant and also a large loss. The appearance of this process is clearly shown by some of the data from table 3 for 66 nylon as plotted in figure 7 . This phenomenon has been observed and reported previously $[1,2,3]$, and is also apparent in figures 5 and 6. 
TABLE 3. Dielectric constants and loss indexes of 66 nylon samples-Continued

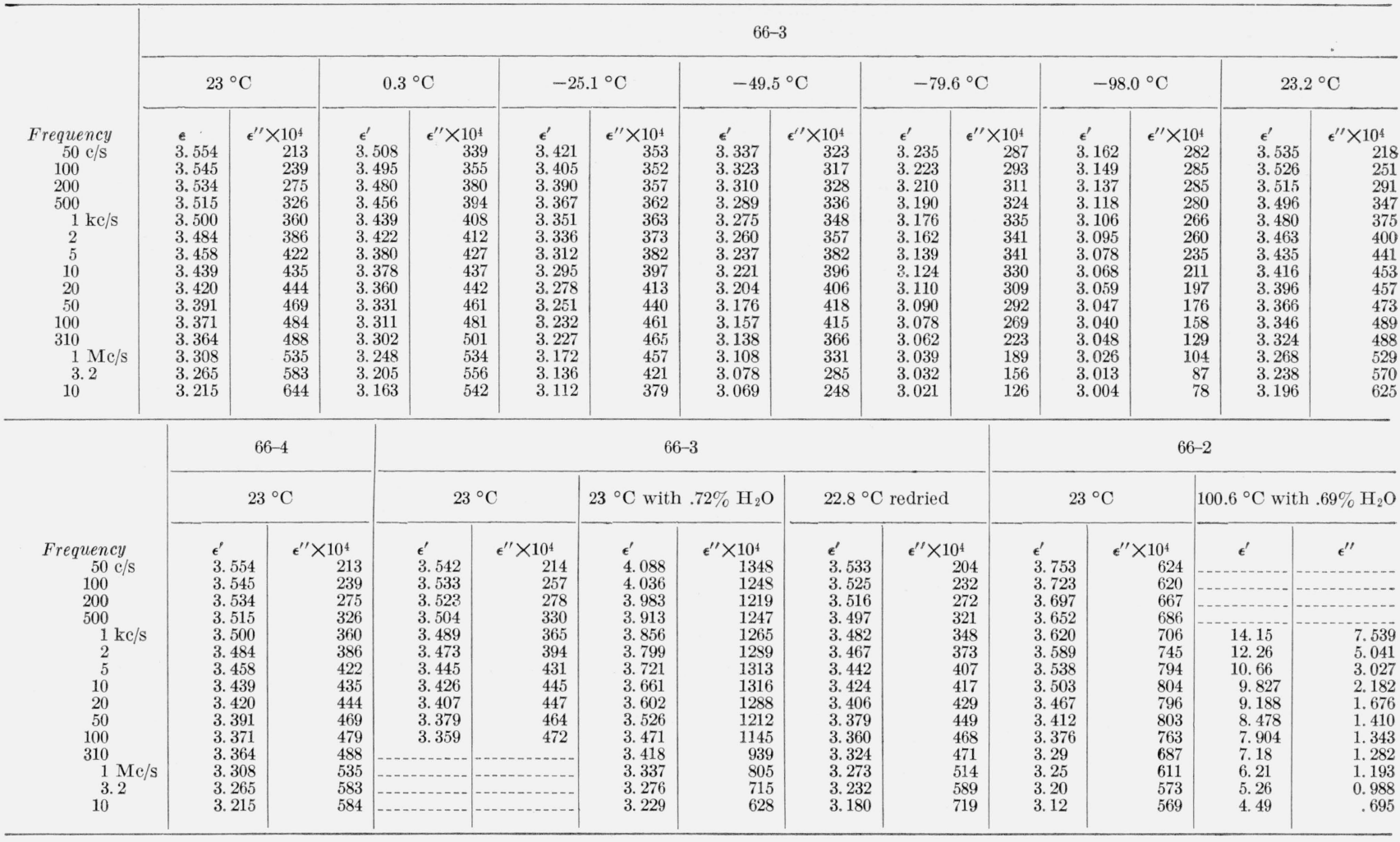

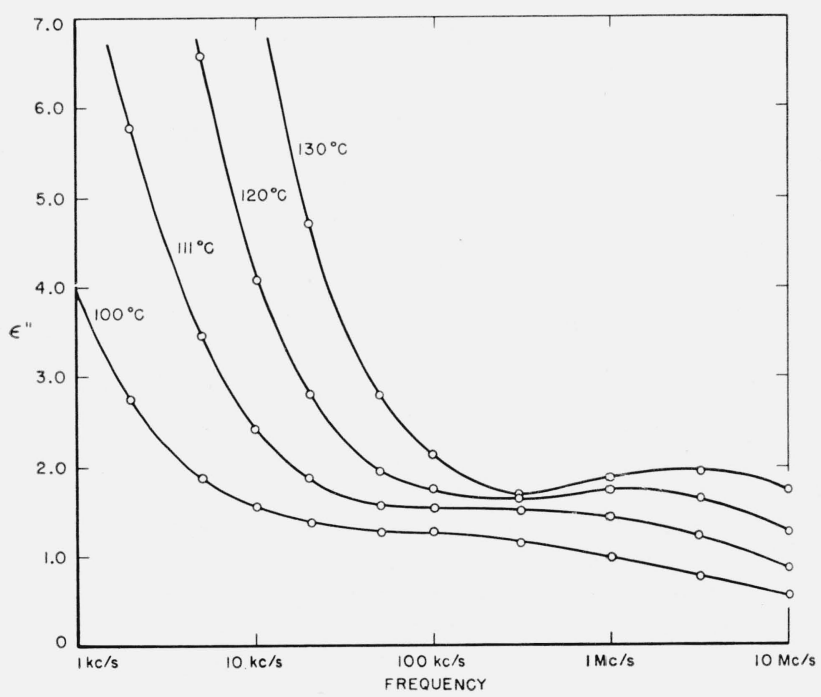

FIgURE 5. Dielectric loss index of 66 nylon as a function of frequency at high temperatures.

The high-temperature dipole relaxation process appears as a maximum at the

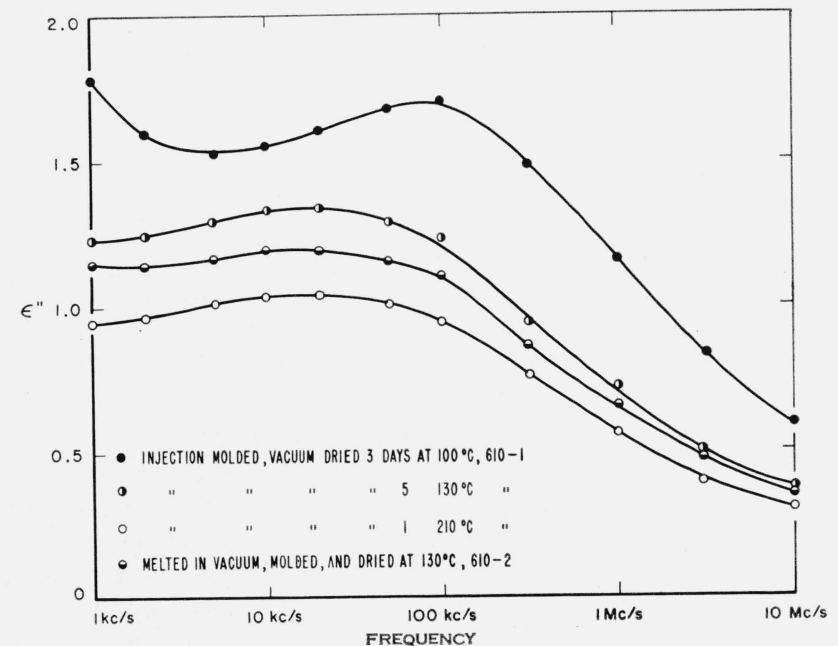

Figure 6. Dielectric loss of 610 nylon at $80^{\circ} \mathrm{C}$ as a function of frequency. 
TABLE 3. Dielectric constants and loss indexes of 66 nylon samples-Continued

\begin{tabular}{|c|c|c|c|c|c|c|c|c|c|c|c|c|c|c|}
\hline \multirow[b]{3}{*}{$\begin{array}{c}\text { Frequency } \\
50 \mathrm{c} / \mathrm{s}\end{array}$} & \multicolumn{14}{|c|}{$66-6$} \\
\hline & \multicolumn{2}{|c|}{$23^{\circ} \mathrm{C}$ not dried } & \multicolumn{2}{|c|}{$100.5^{\circ} \mathrm{C}$ not dried } & \multicolumn{2}{|c|}{$23^{\circ} \mathrm{C}_{\text {dried }}^{\text {vacuum }}$} & \multicolumn{2}{|c|}{ 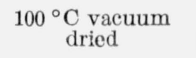 } & \multicolumn{2}{|c|}{$\begin{array}{c}111.0^{\circ} \mathrm{C} \text { vacuum } \\
\text { dried }\end{array}$} & \multicolumn{2}{|c|}{$\begin{array}{c}120.3^{\circ} \mathrm{C} \text { vacuum } \\
\text { dried }\end{array}$} & \multicolumn{2}{|c|}{$\begin{array}{c}130.3^{\circ} \mathrm{C} \text { vacuum } \\
\text { dried }\end{array}$} \\
\hline & $\begin{array}{l}\epsilon^{\prime} \\
3.650\end{array}$ & $\epsilon^{\prime \prime} \times 10^{4}$ & $\epsilon^{\prime}$ & $\epsilon^{\prime \prime}$ & $\begin{array}{l}\epsilon^{\prime} \\
3.600\end{array}$ & $\epsilon^{\prime \prime} \times 16^{4}$ & $\epsilon^{\prime}$ & $\epsilon^{\prime \prime}$ & $\epsilon^{\prime}$ & $\epsilon^{\prime \prime}$ & $\epsilon^{\prime}$ & $\epsilon^{\prime \prime}$ & $\epsilon^{\prime}$ & $\epsilon^{\prime \prime}$ \\
\hline 100 & $\begin{array}{l}0.000 \\
3.628\end{array}$ & 440 & 23.779 & 22.08 & $\begin{array}{l}3.000 \\
3.585\end{array}$ & 322 & 19.66 & 15.95 & & & & & & \\
\hline 200 & 3. 608 & 499 & 18. 924 & 14. 35 & 3. 571 & 372 & 16. 02 & 10.46 & & & & & & \\
\hline 500 & 3.573 & 564 & 14. 956 & 8. 273 & 3.547 & 407 & 13.02 & 6.01 & & & & & & \\
\hline $1 \mathrm{kc} / \mathrm{s}$ & 3. 547 & 610 & 13.055 & 5. 598 & 3. 529 & 430 & 11.58 & 3. 98 & 15.69 & 9.19 & 20.93 & 18.35 & 28.96 & 36.48 \\
\hline 2 & 3. 518 & 668 & 11.758 & 3. 817 & 3. 510 & 465 & 10.52 & 2. 77 & 14.11 & 5. 80 & 17. 23 & 11. 66 & 22.89 & 22. 39 \\
\hline 5 & 3. 472 & 741 & 10.528 & 2.410 & 3.478 & 495 & 9.649 & 1.89 & 11.96 & 3. 476 & 14. 314 & 6.587 & 18. 151 & 12. 22 \\
\hline 10 & 3. 435 & 752 & 9.899 & 1. 891 & 3.457 & 510 & 9.057 & 1.55 & 11. 12 & 2.417 & 12.940 & 4.090 & 15.170 & 7.429 \\
\hline 20 & 3. 402 & 743 & 9.263 & 1. 617 & 3. 434 & 529 & 8.503 & 1. 38 & 10.45 & 1. 887 & 12.020 & 2.814 & 13. 710 & 4. 709 \\
\hline 50 & 3. 355 & 731 & 8. 471 & 1. 477 & 3. 400 & 548 & 7. 784 & 1. 28 & 9.671 & 1. 579 & 11. 153 & 1. 950 & 12.537 & 2. 805 \\
\hline 100 & 3. 322 & 704 & 7.850 & 1.457 & 3. 376 & 571 & 7. 221 & 1. 27 & 9.060 & 1. 547 & 10.558 & 1.748 & 11. 883 & 2.133 \\
\hline 310 & 3. 261 & 584 & 6.992 & 1. 329 & 3. 381 & 566 & 6.463 & 1.14 & 8.138 & 1. 499 & 9. 720 & 1. 624 & 11. 117 & 1. 663 \\
\hline $1 \mathrm{Mc} / \mathrm{s}$ & & & 5. 916 & 1. 151 & 3. 280 & 621 & 5. 573 & 0.985 & 6.917 & 1. 443 & 8.373 & 1. 728 & 9.920 & 1. 880 \\
\hline 3.2 & 3.156 & 737 & 5.102 & .891 & 3. 237 & 658 & 4. 869 & .770 & 5.82 & 1. 216 & 6.970 & 1. 643 & 8.377 & 1. 931 \\
\hline $10^{2.2}$ & 3. 098 & 712 & 4.455 & .691 & 3.160 & 665 & 4. 326 & .566 & 4.91 & 0.893 & 5. 633 & 1. 282 & 6.68 & 1. 737 \\
\hline
\end{tabular}

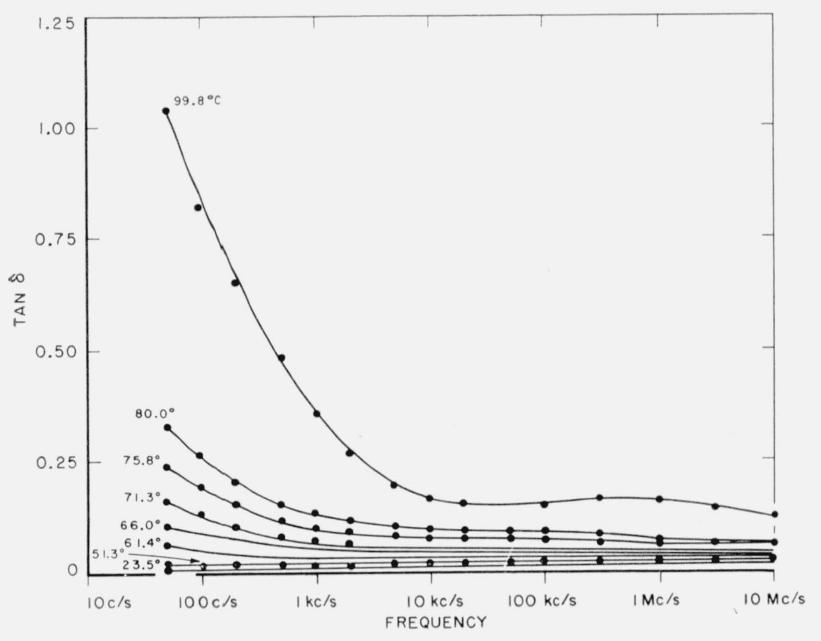

Figure 7. Loss tangent of 66 nylon as a function of frequency.

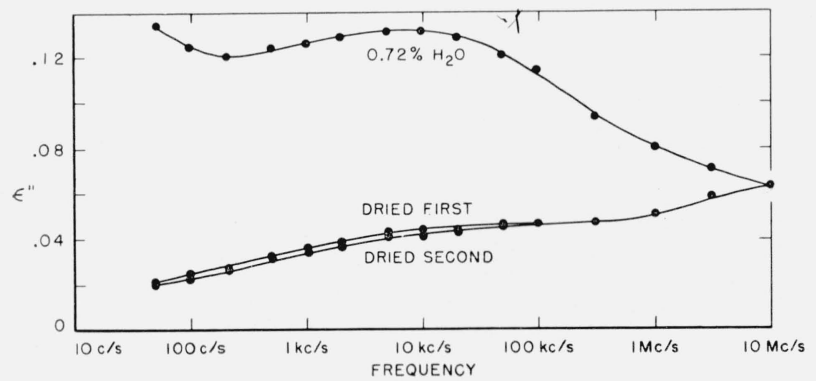

Figure 8. Dielectric loss index of 66 nylon at $23^{\circ} \mathrm{C}$ as a function of frequency.

(3) There is a relaxation process observable at room temperature at about $10 \mathrm{kc} / \mathrm{s}$ in both systems. This process virtually disappeared in the case of 66 nylon after prolonged drying. The relaxation process could be made to reappear after absorption of very small quantities of water, and then to disappear again on drying as shown in figure 8 . In the case of 610 nylon there was no case in which, even after prolonged drying, this relaxation process was completely removed. Rushton and Russell [13] have reported dielectric measurements that showed the pronounced effect that small amounts of water have on this process is 66 nylon, but in no case did they observe an absence of a maximum in their dielectric loss curves.

(4) There is a dipole relaxation process observed between $-100{ }^{\circ} \mathrm{C}$ and $0{ }^{\circ} \mathrm{C}$ in the frequency range studied here. This process, which has not been previously studied by dielectric methods, is present in both polymers, and is characterized by an energy of activation of about $13 \mathrm{kcal}$ per mole of active dipole. Some of the low temperature data for 66 nylon which exhibit the loss peak due to this process are plotted in figure 9 . This relaxation phenomenon is quite probably related to the mechanical relaxation observed in these and other polyamides by Woodward and coworkers [14], Willbourn [15], and Illers and Jenckel [16].

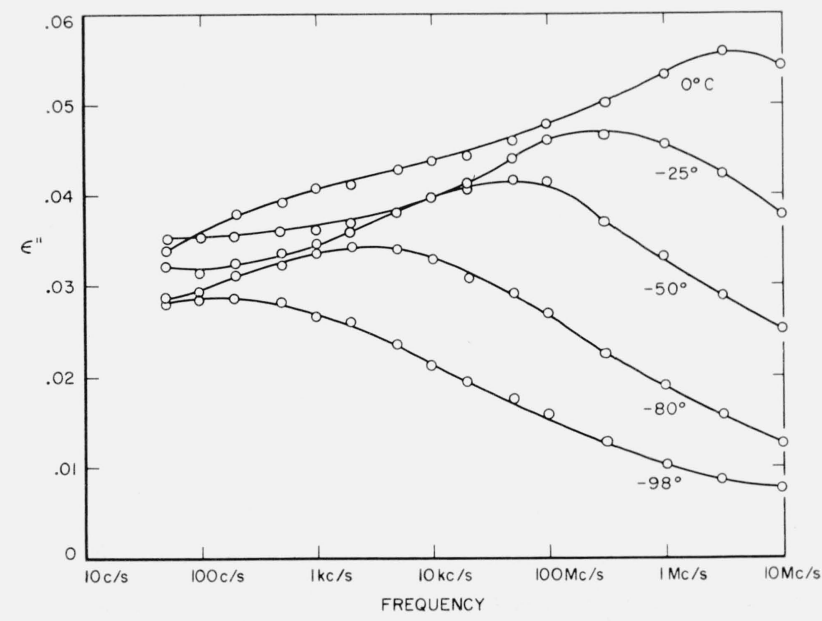

Figure 9. Dielectric loss index of 66 nylon at low temperatures as a function of frequency. 
TABLE 4. Dielectric constants and loss indexes of 610 nylon samples

\begin{tabular}{|c|c|c|c|c|c|c|c|c|c|c|c|c|}
\hline & \multicolumn{6}{|c|}{$610-1$} & \multicolumn{4}{|c|}{ After vacuum drying five days at $130{ }^{\circ} \mathrm{C}$} & \multirow{2}{*}{\multicolumn{2}{|c|}{$\frac{\begin{array}{c}\text { After } 1 \text { day vacuum } \\
\text { drying at } 210^{\circ} \mathrm{C}\end{array}}{80.2{ }^{\circ} \mathrm{C}}$}} \\
\hline & \multicolumn{2}{|c|}{$23{ }^{\circ} \mathrm{C}$} & \multicolumn{2}{|c|}{$80.7^{\circ} \mathrm{C}$} & \multicolumn{2}{|c|}{$100^{\circ} \mathrm{C}$} & \multicolumn{2}{|c|}{$23^{\circ} \mathrm{C}$} & \multicolumn{2}{|c|}{$80.1^{\circ} \mathrm{C}$} & & \\
\hline & $\epsilon^{\prime}$ & $\epsilon^{\prime \prime} \times 10^{4}$ & $\epsilon^{\prime}$ & $\epsilon^{\prime \prime}$ & $\epsilon^{\prime}$ & $\epsilon^{\prime \prime}$ & $\epsilon^{\prime}$ & $\epsilon^{\prime \prime} \times 10^{4}$ & $\epsilon^{\prime}$ & $\epsilon^{\prime \prime}$ & $\epsilon^{\prime}$ & $\epsilon^{\prime \prime}$ \\
\hline $\begin{array}{l}\text { Frequency } \\
50 \mathrm{c} / \mathrm{s}\end{array}$ & 3.665 & 561 & 16.34 & 6.780 & & & 3.558 & 526 & & & & \\
\hline 100 & $\begin{array}{l}5.065 \\
3.640\end{array}$ & $\begin{array}{l}501 \\
627\end{array}$ & $\begin{array}{l}10.34 \\
15.05\end{array}$ & $\begin{array}{l}\text { b. } 480 \\
\text { 4. } 480\end{array}$ & & & $\begin{array}{l}3.558 \\
3.533\end{array}$ & $\begin{array}{l}526 \\
574\end{array}$ & & & & \\
\hline 200 & 3. 614 & 683 & 13.89 & 3.150 & & - & 3.509 & 601 & & & & \\
\hline 500 & 3. 569 & 748 & 12.843 & 2.180 & - & & 3. 468 & 654 & .... & & & \\
\hline $1 \mathrm{kc} / \mathrm{s}$ & 3. 535 & 782 & 12.165 & 1. 780 & 15.990 & $4.570^{-}$ & 3. 438 & 660 & 9.786 & 1. 230 & 8.311 & 0.950 \\
\hline 2 & 3. 501 & 829 & 11.543 & 1. 600 & 14.954 & 3. 070 & 3.410 & 687 & 9.255 & 1. 250 & 7.885 & .970 \\
\hline 5 & 3. 448 & 888 & 10.749 & 1. 530 & 14.046 & 2.030 & 3. 365 & 685 & 8.546 & 1. 300 & 7.307 & 1. 020 \\
\hline 10 & 3. 407 & 895 & 10.078 & 1. 560 & 13.498 & 1. 690 & 3. 336 & 674 & 7.940 & 1. 331 & 6.825 & 1. 040 \\
\hline 20 & 3. 367 & 894 & 9.374 & 1. 610 & 12.865 & 1. 560 & 3. 306 & 645 & 7. 321 & 1. 332 & 6. 338 & 1. 050 \\
\hline 50 & 3. 308 & 879 & 8. 392 & 1. 680 & 12.059 & 1. 640 & 3.265 & 641 & 6.518 & 1. 290 & 5. 702 & 1.010 \\
\hline 100 & 3. 270 & 844 & 7. 583 & 1. 720 & 11.330 & 1. 840 & 3. 236 & 645 & 5. 931 & 1. 240 & 5. 219 & 0.960 \\
\hline 310 & 3. 138 & 677 & 6.480 & 1. 490 & 10.064 & 2. 090 & 3.163 & 621 & 5. 221 & 0.950 & 4.725 & $\begin{array}{r}.752 \\
.750\end{array}$ \\
\hline $1 \mathrm{Mc} / \mathrm{s}$ & 3. 109 & 630 & 5. 337 & 1. 170 & 8. 137 & 2. 200 & 3. 104 & 625 & 4. 563 & .730 & 4. 169 & .570 \\
\hline 3.2 & 3. 094 & 617 & 4. 562 & 0.840 & 6.407 & 1. 840 & 3. 064 & 650 & 4.081 & .510 & 3. 776 & .410 \\
\hline 10 & 3. 009 & 629 & 4.036 & .561 & 5. 142 & 1. 285 & 3. 024 & 671 & 3. 697 & .355 & 3. 510 & .284 \\
\hline
\end{tabular}

$610-2$

\begin{tabular}{|c|c|c|c|c|c|c|c|c|c|c|c|c|c|c|}
\hline & \multicolumn{2}{|c|}{$23^{\circ} \mathrm{C}$} & \multicolumn{2}{|c|}{$80.9^{\circ} \mathrm{C}$} & \multicolumn{2}{|c|}{$+0.2{ }^{\circ} \mathrm{C}$} & \multicolumn{2}{|c|}{$-25.3^{\circ} \mathrm{C}$} & \multicolumn{2}{|c|}{$-50.0^{\circ} \mathrm{C}$} & \multicolumn{2}{|c|}{$-83.1^{\circ} \mathrm{C}$} & \multicolumn{2}{|c|}{$-106.2^{\circ} \mathrm{C}$} \\
\hline & $\epsilon^{\prime}$ & $\epsilon^{\prime \prime} \times 10^{4}$ & $\epsilon^{\prime}$ & $\epsilon^{\prime \prime}$ & $\epsilon^{\prime}$ & $\epsilon^{\prime \prime} \times 10^{4}$ & $\epsilon^{\prime}$ & $\epsilon^{\prime \prime} \times 10^{4}$ & $\epsilon^{\prime}$ & $\epsilon^{\prime \prime} \times 10^{4}$ & $\epsilon^{\prime}$ & $\epsilon^{\prime \prime} \times 10^{4}$ & $\epsilon^{\prime}$ & $\epsilon^{\prime \prime} \times 10^{4}$ \\
\hline $\begin{array}{l}\text { Frequency } \\
50 \mathrm{c} / \mathrm{s}\end{array}$ & 3.324 & 393 & & & 3.311 & 380 & 3. 212 & 364 & 3.119 & 312 & 3. 031 & 283 & 2.943 & 245 \\
\hline 100 & $\begin{array}{l}0.027 \\
3.306\end{array}$ & 429 & & & $\begin{array}{l}3.293 \\
3.293\end{array}$ & 401 & 3. 195 & 357 & 3. 107 & 314 & 3. 020 & 293 & $\begin{array}{l}2.940 \\
2.935\end{array}$ & 244 \\
\hline 200 & 3.287 & 450 & & & 3. 276 & 419 & 3. 180 & 363 & 3. 096 & 324 & 3. 009 & 289 & 2.922 & 240 \\
\hline 500 & 3.257 & 482 & & & 3. 247 & 406 & 3. 157 & 341 & 3. 079 & 317 & 2. 991 & 301 & 2.913 & 228 \\
\hline $1 \mathrm{kc} / \mathrm{s}$ & 3. 236 & 495 & 9.177 & 1. 153 & 3. 229 & 403 & 3. 143 & 329 & 3. 067 & 317 & 2. 978 & 320 & 2.903 & 226 \\
\hline 2 & 3.214 & 504 & 8. 689 & 1. 149 & 3.211 & 400 & 3.128 & 326 & 3. 055 & 317 & 2. 965 & 316 & 2. 896 & 206 \\
\hline 5 & 3.180 & 524 & 8. 035 & 1. 170 & 3.185 & 399 & 3.108 & 329 & 3. 037 & 354 & 2. 951 & 325 & 2.884 & 199 \\
\hline 10 & 3.157 & 514 & 7.499 & 1. 200 & 3. 169 & 399 & 3. 093 & 328 & 3. 024 & 361 & 2. 934 & 312 & 2.877 & 185 \\
\hline 20 & 3.136 & 495 & 6.942 & 1. 200 & 3.152 & 390 & 3.080 & 328 & 3. 010 & 374 & 2. 923 & 285 & 2.870 & 161 \\
\hline 50 & 3. 102 & 495 & 6. 207 & 1. 160 & 3. 127 & 391 & 3. 058 & 350 & 2. 989 & 397 & 2. 907 & 266 & 2.861 & 149 \\
\hline 100 & 3. 082 & 496 & 5. 684 & 1. 120 & 3. 111 & 402 & 3. 043 & 377 & 2. 972 & 405 & 2. 893 & 247 & 2.856 & 140 \\
\hline 310 & 3. 040 & 469 & 5. 060 & 0.870 & 3. 063 & 413 & 2. 987 & 382 & 2. 932 & 351 & 2. 881 & 181 & 2.804 & 110 \\
\hline $1 \mathrm{Mc} / \mathrm{s}$ & 2.978 & 499 & 4.420 & .667 & 2.997 & 446 & 2. 958 & 413 & 2.847 & 318 & 2. 833 & 145 & 2. 789 & 76 \\
\hline 3.2 & 2.939 & 520 & 4. 010 & .480 & 2. 983 & 472 & 2. 954 & 395 & 2. 925 & 280 & 2. 833 & 127 & 2.785 & 72 \\
\hline 10 & 2. 898 & 539 & 3. 654 & .341 & 2. 949 & 470 & 2. 880 & 359 & 2.832 & 238 & 2. 807 & 124 & 2.763 & 73 \\
\hline
\end{tabular}

$610-7$

\begin{tabular}{|c|c|c|c|c|}
\hline \multicolumn{5}{|c|}{ After vacuum drying at $210^{\circ} \mathrm{C}$ for one day } \\
\hline & \multicolumn{2}{|c|}{$23^{\circ} \mathrm{C}$} & \multicolumn{2}{|c|}{$79.5^{\circ} \mathrm{C}$} \\
\hline & $\epsilon^{\prime}$ & $\epsilon^{\prime \prime} \times 10^{4}$ & $\epsilon^{\prime}$ & $\epsilon^{\prime \prime}$ \\
\hline $\begin{array}{l}\text { Frequency } \\
50 \mathrm{c} / \mathrm{s}\end{array}$ & 3. 381 & 399 & 10.164 & 1.466 \\
\hline 100 & 3. 363 & $\begin{array}{l}599 \\
430\end{array}$ & $\begin{array}{r}10.72 \pm \\
9.727\end{array}$ & $\begin{array}{l}1.700 \\
1.210\end{array}$ \\
\hline 200 & 3. 344 & 464 & 9. 288 & 1. 061 \\
\hline 500 & 3. 313 & 478 & 8.791 & 0.990 \\
\hline $1 \mathrm{kc} / \mathrm{s}$ & 3. 291 & 488 & 8. 391 & $\begin{array}{r}.984 \\
\end{array}$ \\
\hline $2^{\mathrm{nU} / \mathrm{S}}$ & 3. 271 & 505 & 7. 938 & .998 \\
\hline$\overline{5}$ & & 504 & 7. 367 & 1. 055 \\
\hline 10 & 3.215 & 497 & 6.899 & 1. 072 \\
\hline 20 & 3. 194 & 483 & 6. 376 & 1. 074 \\
\hline 50 & 3. 163 & 486 & 5. 738 & 1.040 \\
\hline 100 & 3. 141 & 478 & 5. 284 & 0.973 \\
\hline 310 & 3. 067 & 465 & 4. 721 & .766 \\
\hline $1 \mathrm{Mc} / \mathrm{s}$ & 3. 008 & 479 & 4. 212 & .578 \\
\hline 3. 2 & 2. 991 & 510 & 3. 770 & .403 \\
\hline $10^{2}$ & 2. 953 & 581 & 2. 981 & .286 \\
\hline
\end{tabular}

Although further investigation will be necessary before an unambiguous explanation in terms of molecular structure for all of these phenomena can be given, it is possible at this time to rule out some possibilities and to suggest others.

\subsection{High Temperature Dipole Relaxation Process}

The high temperature relaxation process seen in figures 5 and 6 has been extensively studied by Boyd [1] and McCall and Anderson [2], who referred to it as $\alpha^{\prime}$. The results presented here are in no way contradictory to their results. The absence of a clear maximum in the loss index at $80{ }^{\circ} \mathrm{C}$, or even $100{ }^{\circ} \mathrm{C}$, in 66 nylon indicates that the samples studied here were more highly ordered than those used by Boyd. He found a crystallinity of 45 percent while the samples used in this work were 57 percent. These values can be compared, since specific volumes and the same crystallinity scale were used in each case. 
TABLE 4. Dielectric constants and loss indexes of 610 nylon samples-Continued

$610-8$

\begin{tabular}{|c|c|c|c|c|c|c|c|c|c|c|c|c|c|}
\hline \multirow[b]{2}{*}{$\begin{array}{l}\text { Frequency } \\
50 \mathrm{c} / \mathrm{s}\end{array}$} & \multicolumn{2}{|c|}{$23^{\circ} \mathrm{C}$} & \multicolumn{2}{|c|}{$0^{\circ} \mathrm{C}$} & \multicolumn{2}{|c|}{$-26.1^{\circ} \mathrm{C}$} & \multicolumn{2}{|c|}{$-50.1^{\circ} \mathrm{C}$} & \multicolumn{2}{|c|}{$-75.6^{\circ} \mathrm{C}$} & \multicolumn{2}{|c|}{$-100.1^{\circ} \mathrm{C}$} & \multirow{2}{*}{$\frac{80.5^{\circ} \mathrm{C}}{\epsilon^{\prime \prime}}$} \\
\hline & $\epsilon^{\prime}$ & $\epsilon^{\prime \prime} \times 10^{4}$ & $\epsilon^{\prime}$ & $\epsilon^{\prime \prime} \times 10^{4}$ & $\epsilon^{\prime}$ & $\epsilon^{\prime \prime} \times 10^{4}$ & $\epsilon^{\prime}$ & $\epsilon^{\prime \prime} \times 10^{4}$ & $\epsilon^{\prime}$ & $\epsilon^{\prime \prime} \times 10^{4}$ & $\epsilon^{\prime}$ & $\epsilon^{\prime \prime} \times 10^{4}$ & \\
\hline $100 \mathrm{c} / \mathrm{s}$ & $\begin{array}{l}3.429 \\
3.409\end{array}$ & $\begin{array}{l}455 \\
487\end{array}$ & $\begin{array}{l}3.254 \\
3.241\end{array}$ & $\begin{array}{l}364 \\
378\end{array}$ & $\begin{array}{l}3.189 \\
3.173\end{array}$ & $\begin{array}{l}350 \\
348\end{array}$ & $\begin{array}{l}3.120 \\
3.107\end{array}$ & $\begin{array}{l}304 \\
287\end{array}$ & $\begin{array}{l}\text { 3. } 048 \\
\text { 3. } 038\end{array}$ & $\begin{array}{l}241 \\
249\end{array}$ & $\begin{array}{l}2.972 \\
2.962\end{array}$ & $\begin{array}{l}238 \\
238\end{array}$ & \\
\hline 200 & 3. 388 & 504 & 3. 227 & 394 & 3.159 & 346 & 3. 093 & 280 & 3. 028 & $\begin{array}{l}259 \\
252\end{array}$ & 2. 953 & 238 & \\
\hline 500 & 3.355 & 536 & 3. 205 & 399 & 3. 136 & 341 & 3.076 & 281 & 3. 011 & 272 & 2. 938 & 227 & \\
\hline $1 \mathrm{kc} / \mathrm{s}$ & 3. 331 & 543 & 3. 188 & 395 & 3. 123 & 323 & 3.062 & 283 & 2.999 & 282 & 2. 927 & 222 & $1.17 \overline{8}$ \\
\hline 2 & 3.308 & 546 & 3. 174 & 390 & 3. 109 & 317 & 3.050 & 286 & 2.988 & 285 & 2.918 & 221 & 1. 184 \\
\hline 5 & 3.273 & 559 & 3.150 & 398 & 3.089 & 324 & 3. 032 & 312 & 2. 968 & 304 & 2. 904 & 209 & 1. 208 \\
\hline 10 & 3. 248 & 549 & 3. 132 & 392 & 3.075 & 320 & 3.018 & 319 & 2. 954 & 297 & 2. 896 & 188 & 1. 238 \\
\hline 20 & 3.224 & 529 & 3. 116 & 381 & 3.062 & 320 & 3. 004 & 329 & 2. 941 & 285 & 2.888 & 169 & 1. 242 \\
\hline 50 & 3. 192 & 535 & 3. 092 & 393 & 3.041 & 349 & 2.982 & 356 & 2.923 & 276 & 2. 878 & 155 & 1. 203 \\
\hline 100 & 3. 169 & 535 & 3.076 & 393 & 3.026 & 365 & 2.965 & 359 & 2.911 & 257 & 2.871 & 139 & 1. 130 \\
\hline 310 & 3.155 & 503 & 3.035 & 393 & 2. 981 & 374 & 2. 923 & 332 & 2.871 & 221 & 2.847 & 119 & . \\
\hline $1 \mathrm{Mc} / \mathrm{s}$ & 3. 054 & 509 & 2. 987 & 432 & 2.941 & 398 & 2.840 & 313 & 2. 824 & 195 & 2.815 & 97 & 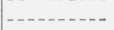 \\
\hline 3. 2 & 3. 030 & 523 & 2. 963 & 474 & 2. 901 & 391 & 2. 859 & 286 & 2. 832 & 171 & 2. 812 & 85 & \\
\hline 10 & 2.986 & 502 & 2. 937 & 494 & 2.886 & 372 & 2. 837 & 240 & 2.821 & 135 & 2. 821 & 120 & \\
\hline
\end{tabular}

$610-9$

\begin{tabular}{|c|c|c|c|c|c|c|c|c|c|c|}
\hline \multirow{3}{*}{$\begin{array}{l}\text { Frequency } \\
50 \mathrm{c} / \mathrm{s}\end{array}$} & \multicolumn{2}{|c|}{$23^{\circ} \mathrm{C}$} & \multicolumn{2}{|c|}{$0^{\circ} \mathrm{C}$} & \multicolumn{2}{|c|}{$-25.3^{\circ} \mathrm{C}$} & \multicolumn{2}{|c|}{$-50.9^{\circ} \mathrm{C}$} & \multicolumn{2}{|c|}{$-75.2^{\circ} \mathrm{C}$} \\
\hline & & $\epsilon^{\prime \prime} \times 10^{4}$ & & $\epsilon^{\prime \prime} \times 10^{4}$ & & $\epsilon^{\prime \prime} \times 10^{4}$ & & $\epsilon^{\prime \prime} \times 10^{4}$ & $\epsilon^{\prime}$ & $\epsilon^{\prime \prime} \times 10^{4}$ \\
\hline & 3. 507 & 454 & 3.345 & 462 & 3. 229 & 446 & 3. 133 & 350 & 3.050 & 272 \\
\hline 100 & 3.488 & 498 & 3. 324 & 485 & 3. 209 & 442 & 3. 119 & 338 & 3.037 & 279 \\
\hline 200 & 3. 466 & 537 & 3.304 & 493 & 3. 191 & 425 & 3.105 & 329 & 3.025 & 287 \\
\hline 500 & 3. 429 & 584 & 3.271 & 515 & 3.165 & 405 & 3. 085 & 325 & 3.006 & 307 \\
\hline $1 \mathrm{ke} / \mathrm{s}$ & 3. 402 & 620 & 3. 249 & 509 & 3. 147 & 385 & 3.071 & 326 & 2. 993 & 315 \\
\hline 2 & 3. 376 & 626 & 3. 226 & 499 & 3. 131 & 376 & 3. 057 & 328 & 2. 979 & 324 \\
\hline 5 & 3. 322 & 641 & 3.195 & 496 & 3. 108 & 376 & 3. 036 & 355 & 2. 957 & 342 \\
\hline 10 & 3. 294 & 644 & 3.173 & 475 & 3. 092 & 372 & 3. 020 & 370 & 2.942 & 338 \\
\hline 20 & 3. 267 & 624 & 3.154 & 458 & 3.077 & 374 & 3. 004 & 379 & 2.927 & 320 \\
\hline 50 & 3. 227 & 616 & 3.125 & 460 & 3. 052 & 404 & 2. 978 & 408 & 2. 905 & 309 \\
\hline 100 & 3. 200 & 610 & 3.105 & 461 & 3. 035 & 428 & 2. 960 & 411 & 2. 892 & 275 \\
\hline 310 & 3. 130 & 565 & 3.054 & 448 & 2. 969 & 431 & 2. 904 & 370 & 2.846 & 239 \\
\hline $1 \mathrm{Me} / \mathrm{s}$ & 3.071 & 577 & 2. 996 & 502 & 2. 924 & 457 & 2.865 & 342 & 2.811 & 206 \\
\hline 3.2 & 3. 029 & 608 & 2. 957 & 533 & 2. 885 & 433 & 2. 832 & 297 & 2. 791 & 175 \\
\hline 10 & 2. 991 & 637 & 2.921 & 540 & 2.851 & 383 & 2.821 & 253 & 2. 797 & 149 \\
\hline
\end{tabular}

The relationship between order, thermal treatment, and the magnitude of the high temperature dipole relaxation process for 610 nylon may be seen by comparison of figure 6 , which shows the loss indexes of several specimens of different degrees of order, and figure 3, which shows the X-ray diffraction intensities of the same specimens. The high temperature loss peak is evidently associated with disordered regions. The specific volume data for these specimens also supports this interpretation.

Sandeman and Keller [8] have pointed out the existence of a disordered crystalline phase in these polyamides. If such a structure exists as a separate phase in the polyamides, it would seem likely that a dipole relaxation phenomenon distinct from that for the even more disordered amorphous phase might be observed. The high temperature relaxation process could be associated with such a disordered crystalline phase. Boyd's observation that cross linking by high energy electron irradiation caused a progressive disappearance of this dielectric relaxation process in 66 nylon, with very little effect on the relaxation time, can more readily be understood in terms of such a phase. It is reasonable to suppose that a few cross links in a disordered crystalline region would more effectively prevent chain rotation than in the amorphous phase. Chemical cross linking in amorphous polymers is known in some instances to raise the glass transition temperature [24]. If this relaxation process were related to such a transition, one might have expected a definite lowering of the frequency of the loss maximum at a given temperature due to cross linking. The data presented in this study do not prove or substantiate the hypothesis that the high temperature relaxation is due to a disordered crystalline region; they do indicate that this relaxation process is definitely not associated with the highly ordered regions of these polyamides, in agreement with both Boyd, and McCall and Anderson.

\subsection{High Temperature, Low Frequency Polarization}

The low frequency polarization process that is evident above about $50{ }^{\circ} \mathrm{C}$ in both 610 and 66 nylon (see, for example fig. 7 for 66 nylon) has a number of interesting features. The very high loss indexes observed at lower frequencies in both polyamides above this temperature cannot be entirely accounted for in terms of $\mathrm{d}$-c conduction attributable to a simple ionic mechanism. This is readily shown by simple computation from the measured d-c resistance. For example, for 66 nylon at $100{ }^{\circ} \mathrm{C}$, the contribution to the loss index due to $\mathrm{d}$-c conduction is approximately 4.0 at $100 \mathrm{c} / \mathrm{s}$, while the observed loss indexes for the various specimens listed in table 3 in this frequency range are between 15.29 and 22.08, depending on the amount of annealing and drying. This point is further demonstrated by the tremendous rise in dielectric constants. It is noteworthy that the highest loss index at $100{ }^{\circ} \mathrm{C}$ and $100 \mathrm{c} / \mathrm{s}$ is observed for specimen $66-6$ before drying (22.08) while the lowest value is for $66-2$ which had been dried 
(15.29). The value for specimen 66-6 closely approached this latter value after drying (15.95).

'The high dielectric constants and loss indexes are evidently not simply due to electrode polarization; if this were the case, the values of dielectric constant and loss would be roughly proportional to sample thickness under comparable conditions. ${ }^{2}$ The present data, which do show considerable sensitivity to thermal history of the samples show no such trend. Compare for example, the high temperature data in table 3 for samples 66-1 and 66-2 in which the ratio of thickness of $66-1$ to $66-2$ is 0.926 . At about $100{ }^{\circ} \mathrm{C}$ and $100 \mathrm{c} / \mathrm{s}$ for example, for 66-1 and 66-2, the dielectric constants were 20.56 and 18.60 respectively. In this case the comparison shows, as do the data at room temperature, that 66-2 was more nearly dry than 66-1. This low frequency polarization, then, must be largely a bulk property of these polyamides. Consideration of the dielectric properties of the two types of nonpolymeric substances mentioned below suggest the reasonableness of this conclusion.

Hoffman and Smyth [17] have described measurements of the dielectric properties of long chain, solid alcohols. A number of these compounds exhibit the interesting property of existing over some temperature range in "rotator" phases. In these rotator phases, the molecules exhibit hindered rotation with consequent dipole orientation. The hydroxyl groups in the crystal structure are arranged in sheets and are joined by hydrogen bonds. In the rotator phases, these hydrogen bonds occasionally break and reform to allow molecular orientation. 'The dielectric constants and losses were unusually high for these materials, and both quantities increased by as much as a factor of four in passing from the liquid to the rotator solid. These losses were interpreted in terms of a large proton drift mobility in the sheets of hydroxyls in the disordered rotator phase. The high dielectric constant was evidently a result of polarization due to a bound anion together with cation (proton) mobility.

Leader and Gormley [18, 19] have reported measurements of a number of amides and $N$-substituted amides in the liquid state. They observed exceedingly high dielectric constants for the $N$-monosubstituted amides: $N$-methylformamide had a dielectric constant of 182.4 at $25{ }^{\circ} \mathrm{C}$ while that of $N$-methylproprionamide was 172.2 at the same temperature. They observed that unsubstituted amides had lower dielectric constants, 110 for formamide and 74 for acetamide. The disubstituted amides had much lower dielectric constants: 36.71 for $N, N$-dimethylformamide and 37.78 for $N$, $N$-dimethylacetamide. It is clear that the polyamides under consideration have structures analogous

2 This is so because electrode polarization would introduce a polarization confined to the immediate area of the electrodes, independent of sample thickness. If this effect were pronounced, it would outweigh the effect of bulk polarization. Thus the measured dielectric constant would equal some capacitance caused by electrode polarization divided by the equivalent vacuum capacitance of the volume occupied by the sample: $\epsilon^{\prime}=C_{p} / C_{0}$. The vacuum capacitance of the volume occupied by the sample: $\epsilon^{\prime}=C_{p} / C_{v}$. The capacitance, due to electrode polarization, $C_{p}$, would be independent of sample thickness; the equivalent vacuum capacitance, $C_{v}$, is inversely proportional to sample thickness. Therefore, if pronounced electrode polarization occurs, the measured dielectric constant
should tend to be proportional to thickness. to those of the monosubstituted amides. It might be expected, therefore, that in the liquid state, the amide groups of polyamides might associate into orientable structures of high dipole moment due to hydrogen bonding similar to those proposed by Leader and Gormley for the monosubstituted amides.

These hypotheses suggested a number of further experiments on the nature of the low frequency polarization mechanism, two of which will be briefly described here. It was thought that a slow relaxation process such as this might be "frozen in" at sufficiently low temperatures, resulting in a very slowly decaying polarization. In effect, it was thought that polyamides should make unusually strong electrets. The classical electret forming treatment was given to some samples: the sample disk was heated up to about $125^{\circ} \mathrm{C}$ and a d-c electric field applied. Then, while maintaining the field, the sample was slowly cooled. Strong electrets were formed in this manner with both 66 and 610 nylon, and the electric field remained for many months when stored at room temperature. On reheating above about $80{ }^{\circ} \mathrm{C}$, a small current could be withdrawn from the specimen over a period of hours, and resulted in the disappearance of the electret field.

It was only after these observations had been made that it was found that Wieder and Kaufman [20] had noted the strong electret forming properties of nylon some years previously.

It is also interesting to note that when a d-c field was applied at elevated temperatures to a specimen on which evaporated gold electrodes had been formed that, although the side tow ards the positive electrode appeared unchanged, the gold on the side toward the negative electrode was loose and readily flaked off. This phenomenon might well be due to the formation of hydrogen gas due to the electrolysis of the current carrying protons.

\subsection{Water-Sensitive, Room Temperature Process}

The relaxation process observed at about $10 \mathrm{kc} / \mathrm{s}$ at room temperature is clearly very sensitive to moisture. In figure 8 , it is evident that, at least for 66 nylon, it is possible to remove enough of the water to cause the relaxation process to be almost completely absent. In the case of the 610 nylon, there was a maximum in the loss index curve for all specimens studied. However, careful drying greatly reduced the magnitude of the maximum in this case also.

It is noteworthy that although exposure of a dried specimen to moisture caused a reappearance of the maximum in 66 nylon, such exposure did not alter the appearance of the high temperature $\alpha^{\prime}$ process in these highly crystalline specimens. (In table 3 there was no loss maximum in sample 66-2 containing 0.69 percent water at $100{ }^{\circ} \mathrm{C}$ although the losses are generally higher than in comparable dry samples. This overall increase in loss is in agreement with the already noted effects of moisture on the low frequency, high temperature polarization process.) Thus, since the measurements of Rushton 
and Russell [13] and of Boyd [1] indicate a loss maximum at room temperature in their driest samples, it seems doubtful that all water had been removed.

The strong hydrogen bonding character of the amide groups in these polymers makes understandable their tenacious absorption of water. This effect is aggravated by the nature of the mechanism of forming these polymers, which results in formation of water as a by-product, and the fact that waterforming end groups (amine and carboxyl groups), remain after polymerization to a finite molecular weight. Thus, chemical equilibrium predicts the presence of small quantities of water which are inversely proportional to the molecular weight of the polyamide. The intrinsic viscosity results indicate that the most severe drving conditions have resulted in a shift of the equilibrium to higher molecular weight and, of course, the removal of some water. However, the fact that this dielectric relaxation process is not due to the end groups themselves but is due to water associated with the polymer, is shown by the fact that it may be made to reappear immediately on absorption of small amounts of water. Hydrolysis to reform end groups under the conditions used for these experiments is very improbable.

The fact that the relaxation process under consideration appeared in all the 610 nylon specimens apparently indicates that none of these were as dry as the 66 nylon. It is also significant that the highest intrinsic viscosity $([\eta]=1.33)$ observed for any of the 610 nylon specimens was significantly lower than that for any of the 66 nylon specimens used in dielectric measurements $([\eta]=1.69)$. Comparison of intrinsic viscosities of different polymers cannot be absolutely quantitative where other information is lacking, but in this case, where the chemical structures are so closely similar, it seems safe to say that the molecular weights of the 610 samples were lower in all instances than the 66 samples used for dielectric measurements.

If one makes certain simple assumptions, it is possible to compute the amount of dispersion in the dielectric constant to be expected from a given quantity of water. Assuming that the water absorbed in the nylon has the same polarizability as in the pure state, for which a dielectric constant of 78 is generally quoted at room temperature (21), then one might expect 0.72 percent of water to increase the low frequency dielectric constant by about 0.56 . The data in table 3 for specimen $66-3$ shows a difference in the dielectric constant of about 0.54 with and without this amount of water at $50 \mathrm{c} / \mathrm{s}$. The difference in the dispersion of the dielectric constants in the frequency range studied, namely $50 \mathrm{c} / \mathrm{s}$ to $10 \mathrm{Mc} / \mathrm{s}$, is 0.506 for the specimen with water and after drying. These figures demonstrate the reasonableness of the proposal that this relaxation process is due to the water-polymer complex. (Pure water shows dielectric dispersion in the microwave region at this temperature.)

This water-sensitive dispersion region is almost certainly closely related to the so called $\beta$ process observed in mechanical measurements of polamides $[14,16]$, as noted earlier. Some of the mechanical data of Illers and Jenckel [16] are shown in figure 10 , together with one dielectric point. In this plot of frequency of loss maximum against reciprocal of absolute temperature, it is evident that there is a correspondence between the mechanical $\beta$ process and the room temperature dielectric loss maximum. It is evident from the dielectric and mechanical measurements that the relaxation process is characteristic of the water-polyamide complex and not of the pure polymer. The opinion has been expressed that a small quantity of swelling agent, such as water in this case, does not result in a new relaxation process but rather modifies processes characteristic of the pure polymer. In this case, such a view is not substantiated, and it is worth pointing out that Scheiber and Mead [22] have reported a similar situation with respect to the effect of water on dielectric dispersion in poly(methyl methacrylate).

\subsection{Low Temperature Relaxation Process}

The low temperature relaxation process observed in these data, as plotted in figure 9 , is evidently related to the mechanical relaxation process observed in the same temperature region $[14,16]$. The plot of the frequency of the maximum loss versus reciprocal of absolute temperature for the 66 nylon data is shown at the right-hand side of figure 10. The activation energy is $13 \mathrm{kcal} / \mathrm{mole}$. Also included in this figure are some of the mechanical relaxation data of Illers and Jenckel, together with those of Woodward and coworkers, on what they have called the $\gamma$ process. It is evident that the dielectric data do not exactly match the mechanical data, the latter having an activation energy of 9 kcal/mole. Further, the displacement of the two sets of data indicate a difference in entropy of activation. To understand these differences, it is first necessary to decide what molecular motions are responsible for this process.

The mechanical relaxation process has been widely attributed to motions of the hydrocarbon portions of the polymer chains. Various arguments have been proposed to support this hypothesis [15]. The present results indicating the existence of a dipolar

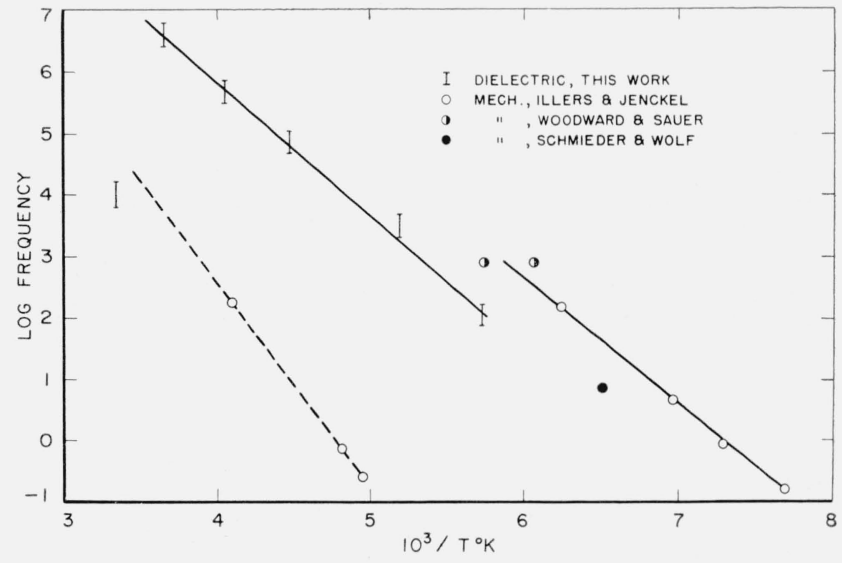

Figure 10. Frequency of loss maximum of 66 nylon as a function of reciprocal temperature.

Water or $\boldsymbol{\beta}$ process indicated by dashed line, low temperature process by solid 
relaxation process necessitate some modification of this view. The difference in entropy of activation as well as the slightly higher energy of activation for the dielectric process suggest a possible mechanism. It seems reasonable that motions of the hydrocarbon segments might be accompanied by motions of the polar amide groups. Because of intermolecular hydrogen bonding, however, such combined motions would be less likely and would also involve a somewhat higher activation energy to break hydrogen bonds. A different entropy for the activated complex would be expected in the two cases. Thus, while the mechanical measurements undoubtedly "see" the relaxation of polar groups to some extent, the much more active motions of the hydrocarbon segments overshadow the process observed in the dielectric measurements.

The hypothesis that this relaxation process is due to motions of molecular end groups can be almost eliminated on the basis of two arguments. First, if such a mechanism were involved, the dielectric and mechanical relaxation processes should coincide at any given temperature and frequency, which is not the case. Second, the magnitude of the polarization associated with this process is not inversely proportional to molecular weight, at least within the limited range covered in the nylon 610 data presented here. However, it is difficult to separate effects of crystallinity and molecular weight. Therefore, the latter argument is not proven, and further work is in progress with other polyamide specimens covering a wider range of molecular weights. It should be pointed out that it is not a simple matter to relate the magnitude of the polarization with the known type and number of polar end groups since in this hydrogen bonded material, the relationship between dipole moment and polarizability cannot be predicted with sufficient accuracy.

\section{Conclusions}

Four relaxation phenomena have been identified in 610 and 66 nylon: (1) Above about $80^{\circ} \mathrm{C}$ a dipolar relaxation process is apparent in both polymers. The earlier suggestion that this process does not occur in highly ordered regions is confirmed by comparison of dielectric, specific volume, and X-ray diffraction data. (2) There is a very-low-frequency polarization characterized by an extremely high loss index and dielectric constant above about $60^{\circ} \mathrm{C}$ in both polyamides. This process, also observed by previous authors, is undoubtedly due to motions of amide group hydrogens involved in intermolecular hydrogen bonds. This process has been found to give rise to electret formation in these polyamides. (3) There is a relaxation process observed at room temperature at about $10 \mathrm{kc} / \mathrm{s}$. This is evidently a result of the presence of water and, in one case, was almost completely absent after prolonged drying. This process has been shown to be the dielectric manifestation of the " $\beta$ process" observed in dynamic mechanical measurements. (4) There is a low temperature relaxation process closely related to the " $\gamma$ process" observed in mechanical measurements.
This process, observed in both polyamides, necessarily involves dipolar motions and probably requires modification of the proposal that the " $\gamma$ process" involves only motions of the hydrocarbon segments in these polymers.

The author is grateful to William P. Harris, who carried out many of the measurements on 66 nylon, and to Benjamin Furst, who carried out the X-ray diffraction measurements.

\section{References}

[1] R. H. Boyd, J. Chem. Phys. 30, 1276, (1959).

[2] D. M. McCall and E. W. Anderson, J. Chem. Phys. 32, 237 (1960).

[3] W. O. Baker and W. A. Yager, J. Amer. Chem. Soc. 64, 2171 (1942).

[4] C. W. Bunn and E. V. Garner, Proc. Roy. Soc. (London) A189, 39 (1947).

[5] W. P. Slichter, J. Poly. Sci. 25, 77 (1958).

[6] G. F. Schmidt and H. A. Stuart, Z. Naturforsch. 13, 222 (1958).

[7] P. W. Allen, Research (London) 5, 492 (1952).

[8] I. Sandeman and A. Keller, J. Poly. Sci. 19, 401 (1956).

[9] A. H. Scott, D. J. Scheiber, A. J. Curtis, J. D. Hoffman, and J. I. Lauritzen, Jr., J. Research NBS, to be published.

[10] J. D. Hoffman and J. J. Weeks, J. Research NBS 60, 465 (1958).

[11] H. W. Starkweather and R. E. Moynihan, J. Poly. Sci. 2\%, 363 (1956).

[12] P. J. Flory, J. Amer. Chem. Soc. 58, 1884 (1936).

[13] E. Rushton and G. Russell, Brit. Elect. and Allied Indust. Research Assoc. Report L/T 355.

[14] A. E. Woodward, J. A. Sauer, C. W. Deeley and D. E. Kline, J. Coll. Sci. 12, 363 (1957).

[15] A. E. Willbourn, Trans. Faraday Soc. 54, 717 (1958).

[16] K. H. Illers and E. Jenckel, I.U.P.A.C. Kurzmitteilungen, Sec. 1: Physik Makromolekular Stoffe, Verlag Chemie, GmbH, Weinheim, (1959), K. H. Illers, Makromoel. Chem. 38, 168, (1960), and K. H. Illers, private communication.

[17] J. D. Hoffman and C. P. Smyth, J. Amer. Chem. Soc. 71, 431 (1949).

[18] G. R. Leader and J. F. Gormley, J. Amer. Chem. Soc. 73, 5731 (1951).

[19] G. R. Leader, J. Amer. Chem. Soc. 73, 856 (1951).

[20] H. H. Wieder and S. Kaufman, J. Appl. Phys. 24, 156 (1953).

[21] C. P. Smyth, Dielectric Behavior and Structure, p. 86, (McGraw-Hill, New York, N.Y., 1955).

[22] D. J. Scheiber and D. J. Mead, J. Chem. Phys. 27, 326 (1957).

[23] A. H. Scott, private communication.

[24] E. B. Baker, P. Auty, and G. J. Ritenour, J. Chem. Phys. 21, 159 (1953).

(Paper 65A3-101) 